\title{
The Fine Structure of Vascular Transfer Cells in Pea Leaf ${ }^{1}$
}

\author{
A. P. Singh \\ Department of Biological Sciences, G. B. Pant University of Agriculture \\ and Technology, Pantnagar, Nainital, U. P., India
}

Received April 28, 1973

The process of solute transfer between the vascular elements and the mesophyll cells has been recognized for many years, however attention has recently been paid to the cells which facilitate this transfer. These cells have rightly been termed 'Transfer Cells' based upon their function (Gunning et al. 1968, Gunning and Pate 1969 a). Transfer cells produce wall ingrowths and, thus, have increased surface area, the magnitude of which varies with the shape and size of these ingrowths. In the last few years several reports have come out dealing with the classification, structure, and function of transfer cells (Gunning et al. 1968, Gunning and Pate 1969, Pate and Gunning 1969, Pate et al. 1969, Pate and Green 1970, O'Brien and Zee 1971, Zee and O'Brien 1971, Yeung and Peterson 1972). Pate and Gunning (1972) have recently given an excellent review on the subject. In the present paper I have attempted to highlight the major cytological features of the xylem and phloem transfer cells in minor veins of pea leaf in relation to their function.

\section{Materials and methods}

Pea seeds (Pisum sativum var. Homesteader) were planted in vermiculite. Plants were grown in growth chambers at $400 \mathrm{ft}-\mathrm{c}$, and about $23^{\circ} \mathrm{C}$ temperature. Leaf samples were fixed in $3 \%$ glutaraldehyde in $0.025 \mathrm{M}$ phosphate buffer, $\mathrm{pH} 6.8$, for two $\mathrm{h}$ at room temperature, and for next $22 \mathrm{~h}$ at $4^{\circ} \mathrm{C}$. Samples were washed in phosphate buffer, post-fixed in $2 \%$ osmium tetroxide, dehydrated in acetone and embedded in araldite. Ultrathin sections were stained with uranyl acetate and lead citrate and examined in a RCA EMU-3H microscope. Thick sections were routinely cut at $1-2 \mu$ and stained with toluidine blue stain for light microscopy.

\section{Observation and discussion}

The classification for transfer cells was proposed by Pate and Gunning (1968). Owing to difficulties encountered in the classification on cytological grounds in pea leaf, I am simply classifying them as xylem and phloem transfer cells, depending upon their association with tracheary and sieve elements. The parenchyma cells occurring in the proximity of vascular tissue and appearing cytologically different from transfer cells are called here vascular parenchyma.

The wall ingrowths of xylem transfer cells appear to be polarized, i.e., they

1 This study was carried out in the electron microscope laboratory of the Department of Biological Sciences, Simon Fraser University, Canada. 


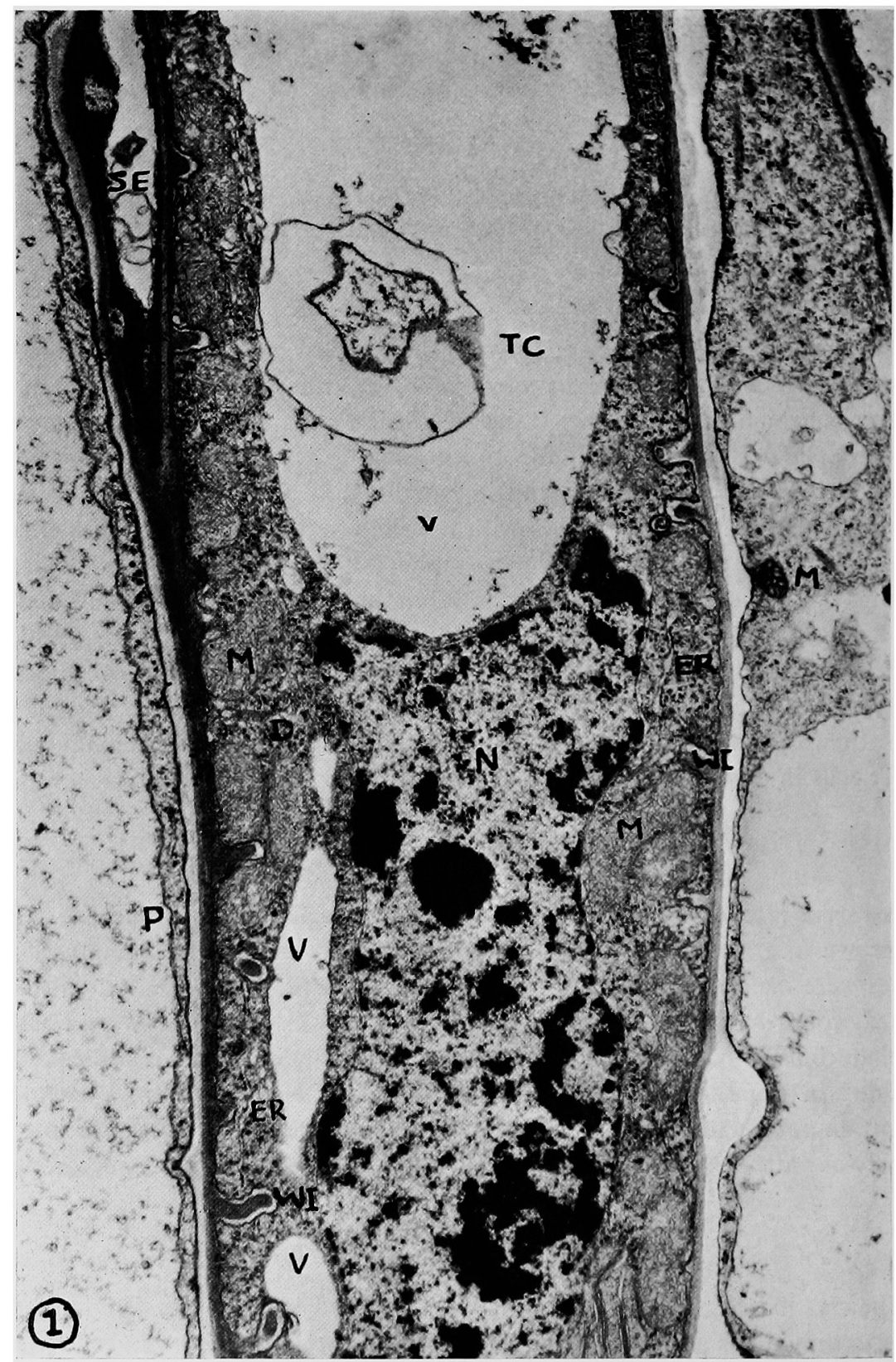

Fig. 1. Paradermal section through parts of transfer cell, sieve element, and vascular parenchyma. Note that the finger-like wall ingrowths are not polarized. Mitochondria are abundant, and ER is peripherally distributed. $\times 12000$. Abbreviations used in the explanation of Figs. 1-7: CHL: Chloroplast, S: Starch grain, CW: Cell wall, SE: Sieve element, D: Dictyosome, SWT: Secondary wall thickening, ER: Endoplasmic reticulum, TC: Transfer cell, M: Mitochondria, TE: Tracheary element, MT: Microtubule, V: Vacuole, N: Nucleus, WF: Wall fibrils, P: Parenchyam, WI: Wall ingrowth, and PD: Plasmodesmata. 
frequently occur on the cell wall abutting tracheary element (Fig. 2). Surprisingly, such polarization is not seen in phloem transfer cells (Fig. 1). This would mean that certain factor (s), which controls the polarization is produced in tracheary elements before the inception of the wall ingrowths. Such factor (s) is either absent from the sieve element or if present, its activity is somehow inhibited. Both types of transfer cells produce papillatetype wall ingrowths, which vary in shape and size, and may occasionally be branched. Plasmalemma is continuous over these ingrowths (Fig. 3). The distinct clear space between the plasmalemma and the wall ingrowth (Fig. 3) may have resulted during the processing of material for electron microscopy. At times, vesicular inclusions are noticed in this space (Figs. 1,3,6). The source of these vesicles is not clear, but they probably contribute to the growth of the wall, although fusion of such vesicles with plasmalemma was not observed. It is possible that the appropriate stages were missed. The role of these vesicles in the wall growth is evidenced by the light coloured material present in them. It should be emphasized that the wall ingrowths help increase the surface of plasmalemma, thus accomodating more carriers, which aid in solute transfer. However, it is not clear whether the wall ingrowth region of the plasmalemma differs from the remaining part in regard to concentration and type of carriers, as it appears remarkably similar in these regions.

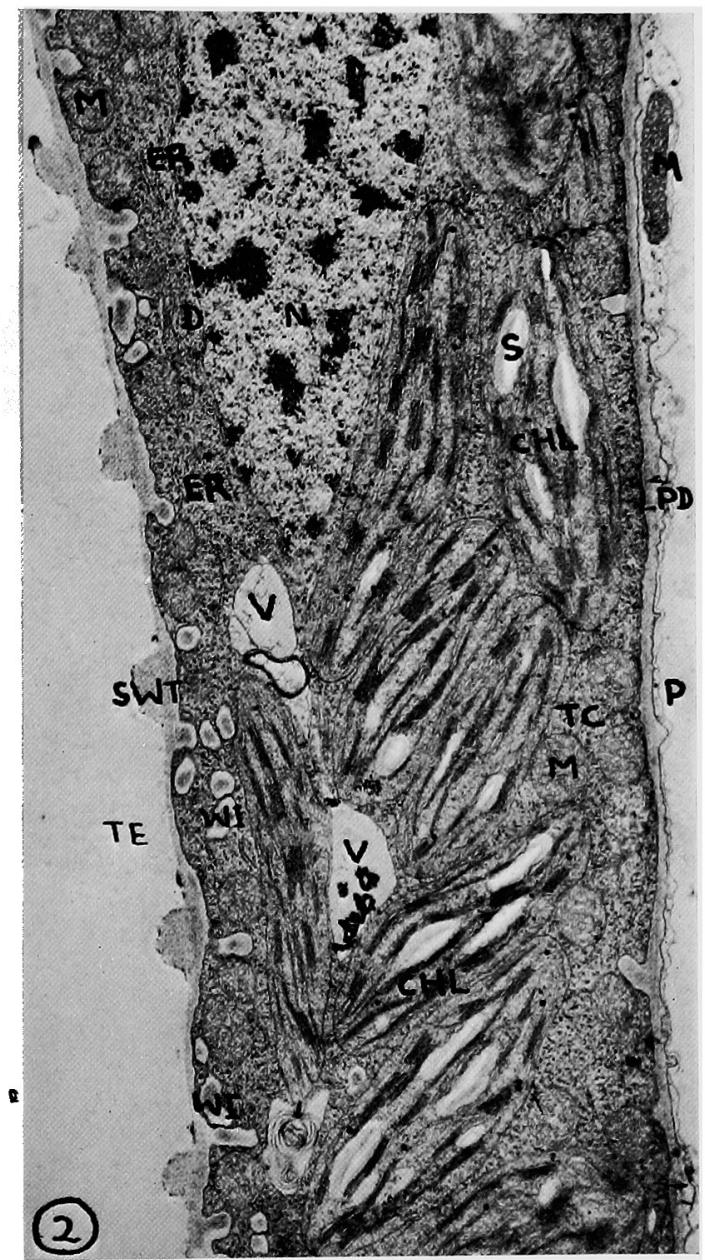

Fig. 2. Paradermal section through parts of transfer cell, tracheary element, and vascular parenchyma. Wall ingrowths appear to be polarized, mitochondria and chloroplasts are abundant, and plasmodesmata traverse the common wall between the transfer cell and vascular parenchyma. $\times 8000$.

The difference in the texture of the ingrowth wall and the wall of tracheary element thickening is apparent (Fig. 7). In the wall ingrowth the wall material is fibrillar and that of the tracheary element thickening is smooth textured. Staining of $1-2 \mu$ thick sections with toluidine blue stain suggests that the wall ingrowth is totally devoid of lignin (see also Zee and O'Brien 1971). The porous nature of the 

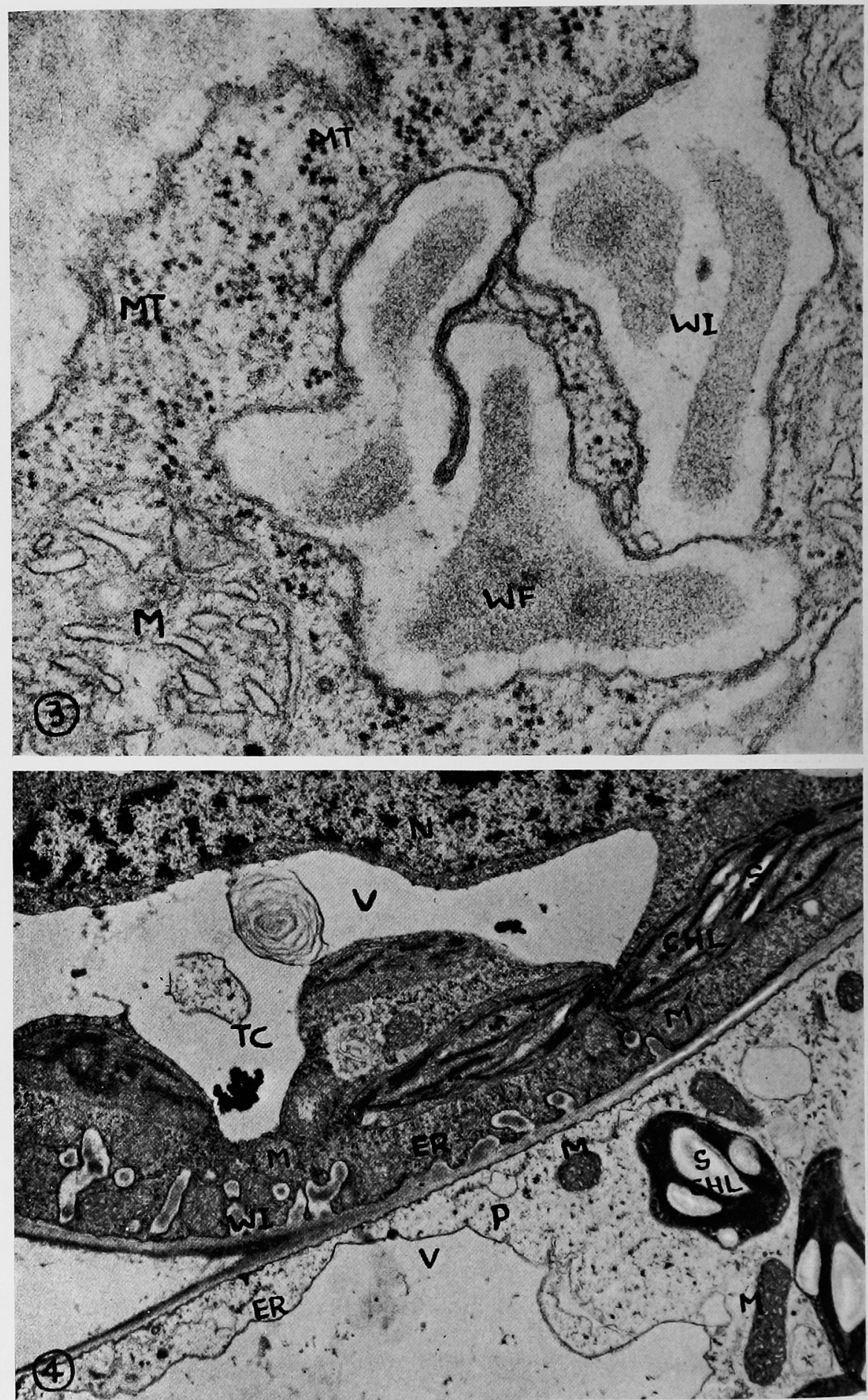

Figs. 3, 4. 3, part of a transfer cell in a grazing section. The branched wall ingrowths have loosely arranged wall fibrils. Microtubules are seen in the proximity of plasmalemma in the peripheral cytoplasm. 4, parts of transfer cell and vascular parenchyma. Note the differences between chloroplasts and mitochondria of the transfer cell and those of vascular parenchyma. Ribosomal density is very high in the transfer cell. Fig. 3, $\times 64000$; Fig. 4, $\times 8000$. 

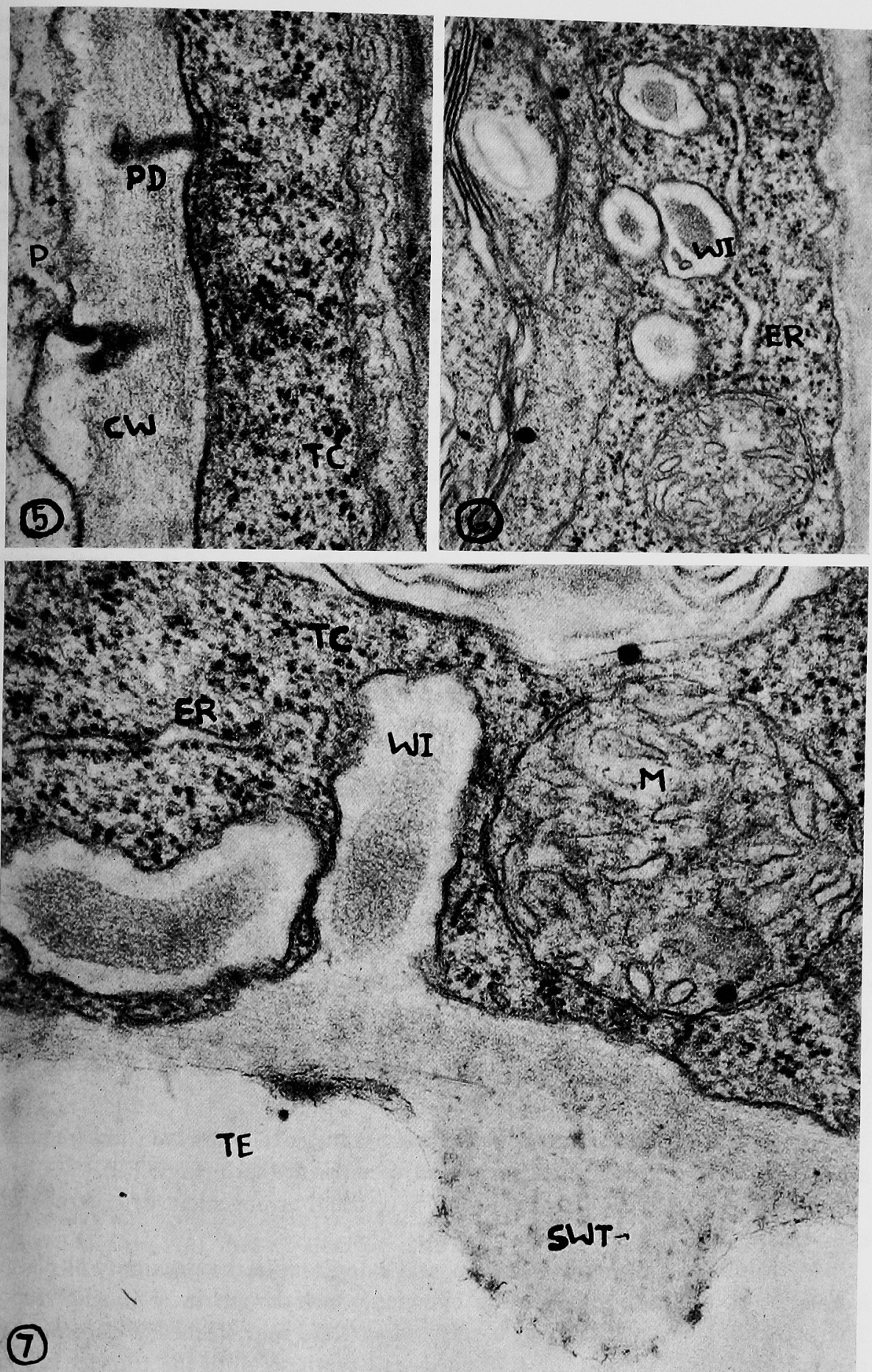

Figs. 5, 6, 7. 5, parts of transfer cell and vascular parenchyma showing plasmodesmatal connection with plasmalemma. 6, part of a transfer cell is shown. ER appears to be in close association with the wall ingrowth. Also, note the vesicular inclusions in the clear space between the plasmalemma and the wall ingrowth. 7, parts of transfer cell and tracheary element are shown. The difference in the wall texture of the wall ingrowth and the secondary wall thickening is apparent.

Fig. 5, ×64000; Fig. 6, ×34000; Fig. 7, $\times 64000$. 
ingrowth wall has been demonstrated by Zee and O'Brien (1971) using lanthanum nitrate tracer, indicating that the cell wall in this region does not provide any resistance to the passage of solutes.

Plasmodesmata traverse the common wall between the sieve element and the transfer cell, between two transfer cells, and between the transfer cell and vascular parenchyma (Fig. 2). But, they are absent from the common wall between the transfer cell and the tracheary element at maturity. Plasmodesmata terminate into plasmalemma (Fig. 5).

Other cytological features of the vascular transfer cell include: high density of ribosomes and rough endoplasmic reticulum (ER), moderately developed chloroplasts, dictyosomes, large number of mitochondria, and microtubules in the peripheral cytoplasm (Figs. 1,2,3). Some of these features have already been described by Gunning and Pate (1969 a), and Zee and O'Brien (1971), and the present findings are in agreement with their observations.

The high density of polyribosomes is indicative of the synthetic activities in transfer cells.

Mitochondria are numerous and have lighter stroma than their counterparts in vascular parenchyma (Figs. 1,2,4). Certainly ATP energy of the mitochondrial respiration is involved in the transfer of solutes. This could be demonstrated through light histochemistry for ATP'ase activity as also suggested by Pate and Gunning (1972).

ER is mainly concentrated in the peripheral zone and at places appears to be in close contact with the plasmalemma over the wall ingrowth (Figs. 1,2,6,7). It is tempting to suggest that the ER may be involved in the growth of wall ingrowths.

Chloroplasts have moderately developed grana with apparently normal stacking of grana thylakoids. They are larger, have lighter stroma and smaller starch grains than their counterparts in the vascular parenchyma (Figs. 2,4). It is interesting to note that the chloroplasts of vascular parenchyma have well developed system of peripheral tubules, which are lacking in the chloroplasts of transfer cells (Fig. 4). It has been suggested that these tubules help in rapid transport of substances into and from the chloroplast (Laetsch 1968, Rosado-Alberio et al. 1968). This should mean that the chloroplasts of transfer cells are less efficient in this regard. Whatever the case, it should be emphasized that transfer cell chloroplasts do carry out some photosynthesis and the energy from photophosphorylation may help in the transfer of solutes.

Usually many small vacuoles occur in the transfer cell, however, less frequently a large central and several small peripheral vacuoles are seen (Figs. 1,2).

Dictyosomes are not infrequent and have usual arrangement of cisternae with end vesicles (Figs. 1,2).

Microtubules are seen in the peripheral cytoplasm in the proximity of plasmalemma. Fig. 3 shows microtubules cut along their length in a grazing section. The presence of microtubules at a stage when sieve and tracheary elements have reached maturity points to the continued wall synthesis until the transfer cell maturity.

Although many reports have been published on the transfer cells during recent 
years, this paper does present additional features of interest. The lack of peripheral tubules in transfer cell chloroplasts, presence of microtubules at a relatively advanced stage, peripheral distribution of ER all add to the already existing cytological features of the transfer cell.

It is not clear at this time what controls initiation and differentiation of the wall ingrowths. It is likely that the neighbouring cell does exercise some influence. At least the polarization of these ingrowths seems to be effected by the factor (s) produced in the neighbouring cell. Plasmalemma synthesis must continue to cope with the growing ingrowths. As pointed out earlier in this paper, the plasmalemma in the region of wall ingrowth does not appear to be any different from the plasmalemma in the rest of the cell, however, the efficiency of these wall ingrowths in the solute exchange should be accounted for. It may be that the plasmalemma, indeed, is identical in all parts of a transfer cell and the rapid solute exchange is brought about only due to the increased surface of the plasmalemma. On the other hand, the possibility of a higher concentration of carriers associated with plasmalemma in the wall ingrowth region can not be ruled out. What role does the chloroplast play in the exchange of solutes, if any? Clearly more work is needed, particularly in enzyme histochemistry and radioisotopy in order to clarify differentiation and certain aspects of transfer cell functioning.

\section{Summary}

The transfer cells in the minor veins of pea leaf develop papillate-type wall ingrowths, which appear to be polarized in the xylem transfer cells. The cytological features include: high density of ribosomes and rough endoplasmic reticulum, moderately developed chloroplasts, dictyosomes, large number of mitochondria, and microtubules in the proximity of plasmalemma. These features are discussed in relation to transfer cell function.

\section{References}

Gunning, B. E. S. and Pate, J. S. 1969 a. "Transfer cells": plant cells with wall ingrowths, specialized in relation to short distance transport of solutes-their occurrence, structure and development. Protoplasma 68: 107-133.

- and -1969 b. Cells with wall ingrowths (transfer cells) in placenta of ferns. Planta (Berl.) 87: 271-274.

-, - and Briarty, L. G. 1968. Specialised "transfer cells" in minor veins of leaves and their possible significance in phloem translocation. J. Cell Biol. 37: 7-12.

-, - and Green, L. N. 1970 a. Transfer cells in the vascular system of stems: taxonomy, association with nodes, and structure. Protoplasma 71: 147-171.

-, - and Milliken, F. F. 1970 b. Function of transfer cells in the nodal regions of stems, particularly in relation to the nutrition of young seedlings. Protoplasma 71: 133-334.

Laetsch, W. M. 1968. Chloroplast specializations in dicotyledons possessing the $\mathrm{C} 4$-dicarboxylic acid pathway of photosynthetic $\mathrm{CO}_{2}$ fixation. Amer. J. Bot. 55: 875-883.

O'Brien, T. P., Zee, S.-Y. and Swift, J. G. 1970. The occurrence of transfer cells in the vascular tissues of the coleoptilar node of wheat. Aust. J. biol. Sci. 23: 709-712.

- and -1971 . Vascular transfer cells in the vegetative nodes of wheat. Aust. J. biol. Sci. 24: 207-217. 
Pate, J. S. and Gunning, B. E. S. 1972. Transfer cells. Ann. Rev. Plant Physiol. 23: 173-196.

Rosado-Alberio, J., Weier, T. E. and Stocking, C. R. 1968. Continuity of the chloroplast membrane systems in Zea mays L. Plant Physiol. 43: 1325-1331.

Yeung, E. C. and Peterson, R. L. 1972. Xylem transfer cells in the rosette plant Hieracium floribundum. Planta (Berl.) 107: 183-188.

Zee, S.-Y. and O'Brien, T. P. 1971. Vascular transfer cells in the wheat spikelet. Aust. J. biol. Sci. 24: 35-49.

- and O'Brien, T. P. 1971. Aleurone transfer cells and other structural features of the spikelet of millet. Aust. J. biol. Sci. 24: 391-395. 